\title{
New controllers and new designs for continuous-time Takagi-Sugeno models
}

\author{
Abdelhafidh Jaadari $^{1,2}$, Thierry Marie Guerra \\ LAMIH UMR CNRS 8201 \\ ${ }^{1}$ University of Valenciennes Hainaut-Cambrésis \\ Valenciennes, France \\ Abdelhafidh.Jaadari; guerra@univ-valenciennes.fr
}

\author{
Antonio Sala \\ ${ }^{2}$ Instituto de Automática e Informática Industrial (AI2) \\ Universitat Politecnica de Valencia \\ Valencia, Spain \\ asala@isa.upv.es
}

\begin{abstract}
This work presents a new non-PDC controller design based on different Lyapunov functions for continuous-time Takagi-Sugeno models. Based on this new control law and on Finsler's lemma a new way to derive LMI constraints problems is presented. Both quadratic and non quadratic Lyapunov functions are under consideration. Some examples show the capability of outperforming existing results without increasing significantly the LMI problems.
\end{abstract}

Keywords: nonquadratic Lyapunov function; quadratic Lyapunov function; linear matrix inequality; Takagi-Sugeno models.

\section{INTRODUCTION}

For more than two decades, Takagi-Sugeno (TS) models [1] have been the subject of an intensive research by the control community, since they can exactly represent a broad class of nonlinear models allowing systematic control and observer design via linear matrix inequalities (LMIs) [3]. LMIs are efficiently solved by convex programming techniques already implemented in commercially available software [6]. Through the sector nonlinearity methodology [2], nonlinear models are rewritten as weighted convex sums of linear ones whose weights capture the nonlinearities into membership functions (MFs) [3]. TS models are combined with different control laws, among which parallel distributed compensation (PDC) is considered a natural option for it reproduces the convex structure of the TS model [4]. Once a TS model and a control law are proposed, the direct Lyapunov method is applied to obtain LMI conditions for control, observer and performance design [3],[36].

Nevertheless, the aforementioned LMI conditions are only sufficient. It may be the case that the choice of Lyapunov function, the way the MFs are included or discarded from the analysis as well as the model construction, seriously restrict the problem feasibility [7],[5]. The last decade witnessed the development of many results aimed to alleviate these drawbacks: different ways to drop the convex sums as to take

\author{
Miguel Bernal \\ Department of Electric and Electronics Engineering \\ Sonora Institute of Technology \\ Ciudad Obregón, Mexico \\ miguel.bernal@itson.edu.mx
}

\author{
Kevin Guelton \\ CReSTIC EA 3804, \\ University of Reims Champagne-Ardenne \\ Reims, France \\ kevin.guelton@univ-reims.fr
}

advantage of the MF information [24],[12],[19],[21]; more inclusive convex models such as the descriptor forms [28],[8] or the polynomial ones [9],[31]; more general Lyapunov functions such as piecewise [10],[11] and fuzzy ones [16],[15].

In the latter direction, numerous fruitful non-quadratic schemes with increasing generality have appeared for discretetime TS models: $k$-sample variations of the Lyapunov function [27], consideration of the model state over several samples [18], improvement of conditions through the Finsler's lemma [27]. So far, continuous-time counterparts of these results have not appeared due to a single obstacle: the time-derivative of the Lyapunov function obliges to deal with the time-derivatives of the MFs which are not easy to cast as a convex problem [13]. Originally, simple bounds of these time-derivatives were used to obtain sufficient conditions which proved to be very conservative and not readily available [14],[32]. Recently, a way to escape from this limitation and provide sound generalizations using a priori known bounds has appeared [26],[22],[23],[33] thus providing an opening to reconsider continuous-time counterparts.

This paper is organized as follows: Section II establishes notation and definitions used throughout this work; section III considers the non-PDC controller design showing that the link between controller and Lyapunov function can be cut in a convenient manner via Finsler's lemma. It is done both for quadratic and non quadratic Lyapunov functions. Section IV gives some examples.

\section{DEFINITIONS AND NotATIONS}

In this work, affine-in-control nonlinear systems of the following form will be considered:

$$
\dot{x}(t)=f(z(t)) x(t)+g(z(t)) u(t)
$$


with $f(\cdot), g(\cdot)$ nonlinear functions, $x(t) \in \mathbb{R}^{n}$ the state vector, $u(t) \in \mathbb{R}^{m}$ the input vector, $z(x(t)) \in \mathbb{R}^{p}$ the premise vector bounded and smooth in a compact set $C \supset \mathbf{0}$.

Let $n l_{j}(\cdot) \in\left[\underline{n l_{j}}, \overline{n l_{j}}\right], j \in\{1, \ldots, p\}$ be the set of bounded nonlinearities of (1) in $C$. Sector nonlinearity approach [2] will be used to construct a TS model of (1). To that end, the following weighting functions are defined

$$
w_{0}^{j}(\cdot)=\frac{\overline{n l_{j}}-n l_{j}(\cdot)}{\overline{n l_{j}}(\cdot)-\underline{n l_{j}}}, \quad w_{1}^{j}(\cdot)=1-w_{0}^{j}(\cdot),
$$

with $j \in\{1, \cdots, p\}$, from which the following MFs are systematically constructed:

$$
h_{i}=h_{1+i_{1}+i_{2} \times 2+\cdots+i_{p} \times 2^{p-1}}=\prod_{j=1}^{p} w_{i_{j}}^{j}\left(z_{j}\right),
$$

with $i \in\left\{1, \ldots, 2^{p}\right\}, i_{j} \in\{0,1\}$. These MFs satisfy the convex sum property $\sum_{i=1}^{r} h_{i}(\cdot)=1, h_{i}(\cdot) \geq 0$ in $C$.

Then, the following TS model is derived with $r=2^{p}$ :

$$
\dot{x}(t)=\sum_{i=1}^{r} h_{i}(z(t))\left(A_{i} x(t)+B_{i} u(t)\right)
$$

where $\left(A_{i}, B_{i}\right), i \in\{1, \ldots, r\}$, are controllable pairs of matrices of proper dimensions. Note that TS model (4) is an exact representation of the nonlinear one (1) in $C$; it is not an approximation.

Certain expressions involving convex sums will be written shortly as follows: $\Upsilon_{z}=\sum_{i=1}^{r} h_{i}(z(t)) \Upsilon_{i}, \Upsilon_{z}^{-1}=\left(\sum_{i=1}^{r} h_{i}(z(t)) \Upsilon_{i}\right)^{-1}$, $\dot{\Upsilon}_{z}^{-1}=\frac{d}{d t}\left(\left(\sum_{i=1}^{r} h_{i}(z(t)) \Upsilon_{i}\right)^{-1}\right)$ and $\Upsilon_{z z}=\sum_{i=1}^{r} \sum_{j=1}^{r} h_{i}(z(t)) h_{j}(z(t)) \Upsilon_{i j}$; A star (*) in a symmetric matrix denotes the transpose of its symmetric element; in a sum it is the transpose of the terms on its left-hand side. In matrix expressions, symbols " $>$ " and " $<$ " stand for positive and negative-definiteness, respectively. When convenient, arguments will be omitted.

MFs will be dropped out from nested convex sums to obtain LMI conditions; they must share at least the same MFs in a double convex sum to use the next relaxation lemma which does not add slack variables:

Relaxation Lemma [24]: Let $\Upsilon_{i j}$ be matrices of proper dimensions. Then $\Upsilon_{z z}<0$ is implied by

$$
\begin{aligned}
& \Upsilon_{i i}<0, \quad \forall i \in\{1, \cdots, r\} \\
& \frac{2}{r-1} \Upsilon_{i i}+\Upsilon_{i j}+\Upsilon_{j i}<0, \quad \forall(i, j) \in\{1, \cdots, r\}^{2}, i \neq j .
\end{aligned}
$$

The following well-known matrix property will play an essential role in obtaining the results presented in this paper:

Finsler's Lemma [34]: Let $x \in \mathbb{R}^{n}, Q=Q^{T} \in \mathbb{R}^{n \times n}$, and $R \in \mathbb{R}^{m \times n}$ such that $\operatorname{rank}(R)<n$; the following expressions are equivalent:
a) $x^{T} Q x<0, \forall x \in\left\{x \in \mathbb{R}^{n}: x \neq 0, R x=0\right\}<0$
b) $\exists X \in \mathbb{R}^{n \times m}: Q+X R+R^{T} X^{T}<0$.

\section{NON-PDC CONTROLLER DESIGN}

A central idea in this paper is to somehow "cut" the link between the Lyapunov function and a non-PDC control law. To that end, quadratic and non-quadratic Lyapunov functions will be considered. This treatment intends to gradually introduce the use of Finsler's Lemma as to suggest the way time-derivatives of the MFs can be handled.

\section{A. Quadratic Lyapunov function}

Consider the following quadratic Lyapunov function candidate with $P=P^{T}>0$

$$
V(x(t))=x(t)^{T} P^{-1} x(t),
$$

and the non-PDC control law

$$
u(t)=F_{z} H_{z}^{-1} x(t),
$$

so as to produce the following closed-loop TS model:

$$
\dot{x}(t)=\left(A_{z}+B_{z} F_{z} H_{z}^{-1}\right) x(t) .
$$

Theorem 1: The TS model (4) under the control law (7) is globally asymptotically stable if $\exists \varepsilon>0$, and matrices $P=P^{T}>0, H_{i}$, and $F_{i}, i=\{1, \ldots, r\}$ of proper dimensions such that (5) holds with $\Upsilon_{i j}=\left[\begin{array}{cc}A_{i} H_{j}+B_{i} F_{j}+(*) & (*) \\ H_{j}-P+\varepsilon\left(A_{i} H_{j}+B_{i} F_{j}\right) & -2 \varepsilon P\end{array}\right]$.

Proof: Consider the Lyapunov function candidate (6); proving that its time-derivative is negative can be written as

$$
\dot{V}=\left[\begin{array}{c}
x \\
\dot{x}
\end{array}\right]^{T}\left[\begin{array}{cc}
0 & P^{-1} \\
P^{-1} & 0
\end{array}\right]\left[\begin{array}{l}
x \\
\dot{x}
\end{array}\right]<0,
$$

which combined with the following expression from (8)

$$
\left[\begin{array}{cc}
A_{z}+B_{z} F_{z} H_{z}^{-1} & -I
\end{array}\right]\left[\begin{array}{c}
x \\
\dot{x}
\end{array}\right]=0
$$

yields, by Finsler's Lemma, the next inequality:

$$
\left[\begin{array}{cc}
0 & P^{-1} \\
P^{-1} & 0
\end{array}\right]+\left[\begin{array}{c}
U \\
W
\end{array}\right]\left[A_{z}+B_{z} F_{z} H_{z}^{-1} \quad-I\right]+(*)<0,
$$


with $U$ and $W$ being matrices of proper dimension. Premultiplying by $\left[\begin{array}{cc}H_{z}^{T} & 0 \\ 0 & P\end{array}\right]$ and post-multiplying by $\left[\begin{array}{cc}H_{z} & 0 \\ 0 & P\end{array}\right]$ allows the following to be obtained:

$$
\left[\begin{array}{cc}
0 & H_{z}^{T} \\
H_{z} & 0
\end{array}\right]+\left[\begin{array}{c}
H_{z}^{T} U \\
P W
\end{array}\right]\left[A_{z} H_{z}+B_{z} F_{z}-P\right]+(*)<0 .
$$

Let $U=H_{z}^{-T}$ and $W=\varepsilon P^{-1}$ with $\varepsilon>0$, so the previous expression renders

$$
\left[\begin{array}{cc}
A_{z} H_{z}+B_{z} F_{z}+(*) & (*) \\
H_{z}-P+\varepsilon\left(A_{z} H_{z}+B_{z} F_{z}\right) & -2 \varepsilon P
\end{array}\right]<0
$$

Applying the Relaxation Lemma to (13) ends the proof.

Important Remark 1: Of course, the problem is not strictly LMI because of the parameter $\varepsilon$. This one is employed in several works concerning linear parameter varying (LPV) systems [34], [35]. It is normally a prefixed value belonging to a family such as: $\varepsilon \in E=\left\{10^{-6}, 10^{-5}, \ldots, 10^{6}\right\}$. This family logarithmically spaced avoids an exhaustive line search. Why is it interesting to use? In [35] the authors showed that for 1000s of LPV models and comparing with numerous results (classical Q approach, Finsler application, and several variants) this way of doing was outperforming in a large way the existing results. Therefore we will follow the same line.

In the next sections parameter $\varepsilon$ (or subscript versions of it) will reappear and preserve the same meaning.

Remark 2: Another important fact is why do we need to introduce the parameter $\varepsilon$ ? This is due to the fact that results in theorem 1 include the ordinary PDC control scheme only if the term $W$ in (12) can be arbitrarily small. To underling this property, consider $H_{z}=P$ in (13), then using Schur complement allows the following expression to be obtained

$$
A_{z} P+B_{z} F_{z}+(*)+\frac{1}{2} \varepsilon\left(A_{z} P+B_{z} F_{z}\right)^{T} P^{-1}\left(A_{z} P+B_{z} F_{z}\right)<0
$$

which for sufficiently small $\varepsilon>0$ is equivalent to the quadratic condition.

\section{B. Quadratic case: introducing constraints}

Consider again the quadratic Lyapunov function candidate (6) together with the control law (7). A way to introduce extra degrees of freedom is to use the control law as another equality constraint via Finsler's lemma. Thus, the open-loop TS model writes:

$$
\dot{x}(t)=A_{z} x(t)+B_{z} u(t) .
$$

Theorem 2: The TS model (15) under the control law (7) is globally asymptotically stable if it exists $\varepsilon>0$ and matrices $P=P^{T}>0, H_{i}$, and $F_{i}, i=\{1, \ldots, r\}$ of proper dimensions such that (5) holds with

$$
\Upsilon_{i j}=\left[\begin{array}{ccc}
A_{i} H_{j}+B_{i} F_{j}+(*) & (*) & (*) \\
H_{j}-P+\varepsilon\left(A_{i} H_{j}+B_{i} F_{j}\right) & -2 \varepsilon P & (*) \\
\varepsilon F_{j} & 0 & -2 \varepsilon I
\end{array}\right]<0 .
$$

Proof: Consider the Lyapunov function candidate (6); proving that its time-derivative is negative can be written as

$$
\dot{V}=\left[\begin{array}{c}
x \\
\dot{x} \\
u
\end{array}\right]^{T}\left[\begin{array}{ccc}
0 & P^{-1} & 0 \\
P^{-1} & 0 & 0 \\
0 & 0 & 0
\end{array}\right]\left[\begin{array}{l}
x \\
\dot{x} \\
u
\end{array}\right]<0
$$

which combined with the following expressions from (15) and (7):

$$
\left[\begin{array}{ccc}
A_{z} & -I & B_{z} \\
F_{z} H_{z}^{-1} & 0 & -I
\end{array}\right]\left[\begin{array}{l}
x \\
\dot{x} \\
u
\end{array}\right]=0
$$

yields, by Finsler's Lemma, the next inequality:

$\left[\begin{array}{ccc}0 & P^{-1} & 0 \\ P^{-1} & 0 & 0 \\ 0 & 0 & 0\end{array}\right]+\left[\begin{array}{cc}U_{1} & V_{1} \\ U_{2} & V_{2} \\ U_{3} & V_{3}\end{array}\right]\left[\begin{array}{ccc}F_{z} H_{z}^{-1} & 0 & -I \\ A_{z} & -I & B_{z}\end{array}\right]+(*)<0$

with $U_{i}$ and $V_{i}, i=\{1, \ldots, 3\}$ being matrices of proper dimension. Pre-multiplying and post-multiplying by $\left[\begin{array}{ccc}H_{z}^{T} & 0 & 0 \\ 0 & P & 0 \\ 0 & 0 & I\end{array}\right]$ and $\left[\begin{array}{ccc}H_{z} & 0 & 0 \\ 0 & P & 0 \\ 0 & 0 & I\end{array}\right]$ gives:

$\left[\begin{array}{ccc}0 & H_{z}^{T} & 0 \\ H_{z} & 0 & 0 \\ 0 & 0 & 0\end{array}\right]+\left[\begin{array}{cc}H_{z}^{T} U_{1} & H_{z}^{T} V_{1} \\ P U_{2} & P V_{2} \\ U_{3} & V_{3}\end{array}\right]\left[\begin{array}{ccc}F_{z} & 0 & -I \\ A_{z} H_{z} & -P & B_{z}\end{array}\right]+\left(^{*}\right)<0$

Let $U_{1}=H_{z}^{-T} B_{z}, U_{2}=\varepsilon P^{-1} B_{z}, U_{3}=\varepsilon I, V_{1}=H_{z}^{-T}$, $V_{2}=\varepsilon P^{-1}$ and $V_{3}=0$ with $\varepsilon>0$ so the previous expression renders:

$$
\left[\begin{array}{ccc}
A_{z} H_{z}+B_{z} F_{z}+\left(^{*}\right) & (*) & (*) \\
H_{z}-P+\varepsilon\left(A_{z} H_{z}+B_{z} F_{z}\right) & -2 \varepsilon P & (*) \\
\varepsilon F_{z} & 0 & -2 \varepsilon I
\end{array}\right]<0
$$

Applying the Relaxation Lemma to (20) ends the proof.

The same discussion as remarks $1 \& 2$ holds in this case. This last result will be extended to a non-quadratic Lyapunov function in the next part.

\section{Non-quadratic Lyapunov function}

Consider the following non-quadratic Lyapunov function candidate with $P_{z}=\sum_{i=1}^{r} h_{i}(z(t)) P_{i}, P_{i}=P_{i}^{T}>0$ : 


$$
V(x(t))=x(t)^{T} P_{z}^{-1} x(t),
$$

and the non-PDC control law

$$
u(t)=F_{z} H_{z}^{-1} x(t),
$$

The corresponding closed-loop TS model is:

$$
\dot{x}(t)=A_{z} x(t)+B_{z} u(t) .
$$

Theorem 3: The TS model (4) under the control law (22) is locally asymptotically stable according to its initial conditions in the outermost Lyapunov level included in the region $R=\left\{x \in \mathbb{R}^{n},\|x\| \leq \lambda_{x}\right\}$, if it exists $\varepsilon>0$ and matrices $P_{j}=P_{j}^{T}>0, S_{j}=S_{j}^{T}>0, H_{j}, F_{j}, j=\{1, \ldots, r\}$ of proper dimensions such that (5) holds with

$$
\begin{aligned}
\Upsilon_{i j} & =\left[\begin{array}{cccc}
A_{i} H_{j}+B_{i} F_{j}+(*) & (*) & (*) & (*) \\
H_{j}-P_{j}+\varepsilon\left(A_{i} H_{j}+B_{i} F_{j}\right) & -2 \varepsilon P_{j} & 0 & 0 \\
\varepsilon F_{j} & 0 & -2 \varepsilon I & 0 \\
\varepsilon H_{j} & 0 & 0 & -\varepsilon P_{j}
\end{array}\right] \\
\Psi_{i j}^{k} & =\left[\begin{array}{cc}
\varphi_{k}\left(H_{j}+H_{j}^{T}-S_{j}\right) & (*) \\
A_{i} H_{j}+B_{i} F_{j} & I
\end{array}\right], I<\varphi_{k} S_{i}
\end{aligned}
$$

and $\quad-\sum_{i=1}^{r} \sum_{k=1}^{p}(-1)^{d_{i}^{\gamma}} \beta_{k}\left(P_{g_{1}(i, k)}-P_{g_{2}(i, k)}\right) \leq \varepsilon P_{j}$

hold with $\varphi_{k}=\frac{2 \beta_{k}}{\lambda_{k}^{2}+\lambda_{x}^{2}}$ and:

$\gamma-1=d_{r}^{\gamma}+d_{r-1}^{\gamma} \times 2+\cdots+d_{1}^{\gamma} \times 2^{r-1}, \gamma=\left\{0, \ldots, 2^{r}-1\right\}$.

Proof: Consider the Lyapunov function candidate (21); in order to guarantee that its time-derivative is negative the following condition must hold:

$$
\dot{V}=\left[\begin{array}{c}
x \\
\dot{x} \\
u
\end{array}\right]^{T}\left[\begin{array}{ccc}
\dot{P}_{z}^{-1} & P_{z}^{-1} & 0 \\
P_{z}^{-1} & 0 & 0 \\
0 & 0 & 0
\end{array}\right]\left[\begin{array}{c}
x \\
\dot{x} \\
u
\end{array}\right]<0,
$$

which combined with the equality constraint (17) yields, by a similar procedure of that employed in theorem 2, the next inequality with $\mathcal{E}>0$ :

$$
\left[\begin{array}{ccc}
H_{z}^{T} \dot{P}_{z}^{-1} H_{z}+A_{z} H_{z}+B_{z} F_{z}+(*) & (*) & (*) \\
H_{z}-P_{z}+\varepsilon\left(A_{z} H_{z}+B_{z} F_{z}\right) & -2 \varepsilon P_{z} & (*) \\
\varepsilon F_{z} & 0 & -2 \varepsilon I
\end{array}\right]<0
$$

In order to deal with $\dot{P}_{z}^{-1}$, consider $Q_{z}=\varepsilon^{-1} P_{z}$ under the following relationship:

$$
\dot{P}_{z}^{-1} \leq Q_{z}^{-1},
$$

Thus, by Schur complement it can be taken into account to guarantee (26) if

$$
\left[\begin{array}{cccc}
A_{z} H_{z}+B_{z} F_{z}+(*) & (*) & (*) & (*) \\
H_{z}-P_{z}+\varepsilon\left(A_{z} H_{z}+B_{z} F_{z}\right) & -2 \varepsilon P_{z} & 0 & 0 \\
\varepsilon F_{z} & 0 & -2 \varepsilon I & 0 \\
\varepsilon H_{z} & 0 & 0 & -\varepsilon P_{z}
\end{array}\right]<0
$$

The previous expression is a double convex-sum of MFs $h_{i}(\cdot)$ and $h_{j}(\cdot), i, j \in\{1, \ldots, r\}$. Thus, the Relaxation Lemma can be applied to (28).

Considering the definition of $Q_{z}$ above and the property $P_{z} \dot{P}_{z}^{-1} P_{z}=-\dot{P}_{z}$, it follows that (27) can be rewritten as:

$$
P_{z} \dot{P}_{z}^{-1} P_{z} \leq P_{z} \varepsilon P_{z}^{-1} P_{z} \Leftrightarrow-\dot{P}_{z} \leq \varepsilon P_{z} .
$$

It has been shown in [22] that:

$$
\dot{P}_{z}=\sum_{j=1}^{r} \sum_{k=1}^{p} h_{j} \frac{\partial w_{0}^{k}}{\partial z_{k}}\left(P_{g_{1}(j, k)}-P_{g_{2}(j, k)}\right) \dot{z}_{k}
$$

With $g_{1}(j, k)=\left\lfloor(j-1) / 2^{p+1-k}\right\rfloor \times 2^{p+1-k}+1+(j-1) \bmod 2^{p-k}$ and $g_{2}(j, k)=g_{1}(j, k)+2^{p-k},\lfloor\cdot\rfloor$ standing for the floor function. Note that:

$$
\dot{z}_{k}=\frac{\partial z_{k}(t)^{T}}{\partial x(t)}\left(A_{z} H_{z}+B_{z} F_{z}\right) H_{z}^{-1} x(t)
$$

Then as in [37], we have to find a way to obtain LMI constraints in order to fulfill:

$$
\left|\frac{\partial w_{0}^{k}}{\partial z_{k}}\left(\frac{\partial z_{k}}{\partial x}\right)^{T}\left(A_{z} H_{z}+B_{z} F_{z}\right) H_{z}^{-1} x\right| \leq \beta_{k}
$$

considering that we know a priori only the bounds:

$$
\left\|\frac{\partial w_{0}^{k}}{\partial z_{k}}\left(\frac{\partial z_{k}}{\partial x}\right)^{T}\right\| \leq \lambda_{k},\|x\| \leq \lambda_{x}
$$

Important remark 3: as $\dot{h}_{i}(z)=\left(\frac{\partial h_{i}}{\partial z}\right)^{T}\left(\frac{\partial z_{k}}{\partial x}\right) \dot{x}$ it implies that $\dot{h}_{i}(z)=f\left(z, x, u, \frac{\partial h_{i}}{\partial x}\right)$, i.e. it is dependent on the control. It implies that the works inducing $\left\|\dot{h}_{i}(z)\right\| \leq \phi_{i}[14]$, [30], [32] CANNOT prove the stabilization excepted A POSTERIORI. This is a major drawback of all these methods. Whereas (33) is perfectly known, thus local stabilization conditions will be obtained in the outermost level in $R=\left\{x \in \mathbb{R}^{n},\|x\| \leq \lambda_{x}\right\}$.

After some manipulation as in [37], considering $S_{z}>0,(32)$ is ensured if:

$$
\left[\begin{array}{cc}
S_{z}^{-1 / 2} & 0 \\
0 & \left(A_{z} H_{z}+B_{z} F_{z}\right) H_{z}^{-1} S_{z}^{1 / 2}
\end{array}\right]^{T} \times(*)<\varphi_{k} I
$$


with $\varphi_{k}=\frac{2 \beta_{k}}{\lambda_{k}^{2}+\lambda_{x}^{2}}$. Thus first row of (34) leads directly to $I<\varphi_{k} S_{z}$, whereas second gives after congruence with $S_{z}^{-1 / 2}$ :

$$
H_{z}^{-T}\left(A_{z} H_{z}+B_{z} F_{z}\right)^{T}\left(A_{z} H_{z}+B_{z} F_{z}\right) H_{z}^{-1}<\varphi_{k} S_{z}^{-1}
$$

Or equivalently:

$$
\left(A_{z} H_{z}+B_{z} F_{z}\right)^{T}\left(A_{z} H_{z}+B_{z} F_{z}\right)<\varphi_{k} H_{z}^{T} S_{z}^{-1} H_{z}
$$

Recalling that for $S_{z}>0: H_{z}^{T} S_{z}^{-1} H_{z} \geq H_{z}+H_{z}^{T}-S_{z}$, (36) is satisfied if:

$$
\left(A_{z} H_{z}+B_{z} F_{z}\right)^{T}\left(A_{z} H_{z}+B_{z} F_{z}\right)<\varphi_{k}\left(H_{z}^{T}+H_{z}-S_{z}\right)
$$

And applying a Schur complement on (37) gives the result of theorem3.

Recalling the property:

$$
Y+\gamma_{k} X \leq 0 \Leftarrow\left\{\begin{array}{l}
Y+\lambda_{k} \times X \leq 0 \\
Y-\lambda_{k} \times X \leq 0
\end{array},\left|\gamma_{k}\right| \leq \lambda_{k}\right.
$$

Thus, (29) holds if conditions (24) hold, which concludes the proof.

Remark 4: Results in theorem 3 include the ordinary PDC control scheme with quadratic Lyapunov function. It can be shown with Schur complement and considering $P_{i}=P$ $i \in\{1, \ldots, r\}$ and $\varepsilon$ arbitrarily small.

\section{EXAMPLES}

Example 1: Consider the nonlinear model:

$\dot{x}(t)=\left[\begin{array}{cc}a+b\left(x_{1}^{2}+x_{2}^{2}\right) & -1 \\ x_{1}^{2}+2 & c+d\left(x_{1}^{2}+x_{2}^{2}\right)\end{array}\right] x(t)+\left[\begin{array}{c}1 \\ -x_{2}^{2}\end{array}\right] u(t)$

with $a=-0.2363, b=0.0985, c=-0.7097$, and $d=0.3427$.

The following TS model can be constructed from (39) in the compact set $C=\left\{x:\left|x_{1}\right| \leq 1,\left|x_{2}\right| \leq 2\right\}$ :

$$
\begin{aligned}
\dot{x}(t) & =\sum_{i=1}^{4} h_{i}(z(t))\left(A_{i} x(t)+B_{i} u(t)\right) \\
y(t) & =\sum_{i=1}^{4} h_{i}(z(t)) C_{i} x(t)
\end{aligned}
$$

with $A_{1}=\left[\begin{array}{cc}-a+5 b & -1 \\ 3 & -c+5 d\end{array}\right] \quad, \quad A_{2}=\left[\begin{array}{cc}-a+b & -1 \\ 3 & -c+d\end{array}\right]$, $A_{3}=\left[\begin{array}{cc}-a+4 b & -1 \\ 2 & -c+4 d\end{array}\right], A_{4}=\left[\begin{array}{cc}-a & -1 \\ 2 & -c\end{array}\right], B_{1}=B_{3}=\left[\begin{array}{c}1 \\ -4\end{array}\right]$, $B_{2}=B_{4}=\left[\begin{array}{l}1 \\ 0\end{array}\right], i=1, \ldots, 4, z_{1}=x_{1}^{2}, \quad z_{2}=x_{2}^{2}, \quad w_{0}^{1}=z_{1}$, $w_{0}^{2}=\frac{z_{2}}{4}, w_{1}^{1}=1-w_{0}^{1}, w_{1}^{2}=1-w_{0}^{2}, h_{1}=w_{0}^{1} w_{0}^{2}, h_{2}=w_{0}^{1} w_{1}^{2}$, $h_{3}=w_{1}^{1} w_{0}^{2}, h_{4}=w_{1}^{1} w_{1}^{2}$. Recall that TS model (40) exactly represents (39) in $C$.

Using theorem 2 with $\varepsilon=1$, a non-PDC controller of the form (7) can be found via a quadratic Lyapunov function (6). The gains and Lyapunov matrix are given by

$$
\begin{aligned}
H_{1} & =\left[\begin{array}{ll}
0.5147 & 0.3107 \\
1.2469 & 2.7004
\end{array}\right], F_{1}=\left[\begin{array}{l}
0.4472 \\
1.7071
\end{array}\right]^{T}, H_{2}=\left[\begin{array}{cc}
0.3894 & -0.8823 \\
0.9513 & 2.1158
\end{array}\right], \\
F_{2} & =\left[\begin{array}{ll}
0.2136 \\
1.5175
\end{array}\right]^{T}, H_{3}=\left[\begin{array}{cc}
0.8551 & 0.5411 \\
1.39 & 2.8006
\end{array}\right], F_{3}=\left[\begin{array}{c}
0.3892 \\
1.6472
\end{array}\right]^{T}, \\
H_{4} & =\left[\begin{array}{ll}
0.7453 & -0.7108 \\
1.1077 & 2.2548
\end{array}\right], F_{4}=\left[\begin{array}{l}
0.1797 \\
1.5333
\end{array}\right]^{T}, P=\left[\begin{array}{ll}
0.8085 & 0.0367 \\
0.0367 & 4.6949
\end{array}\right] .
\end{aligned}
$$

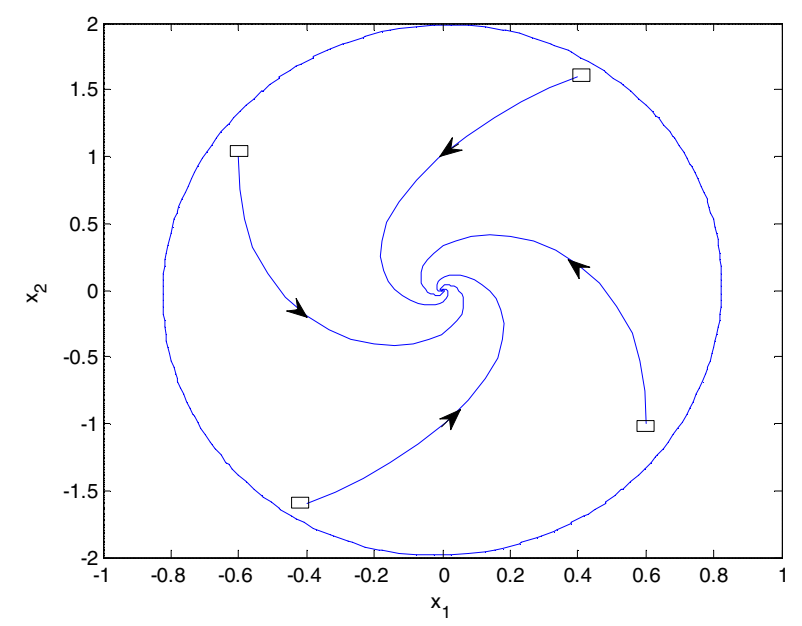

Figure 1. Stabilization region with quadratic Lyapunov function and nonPDC controller under theorem 2 scheme.

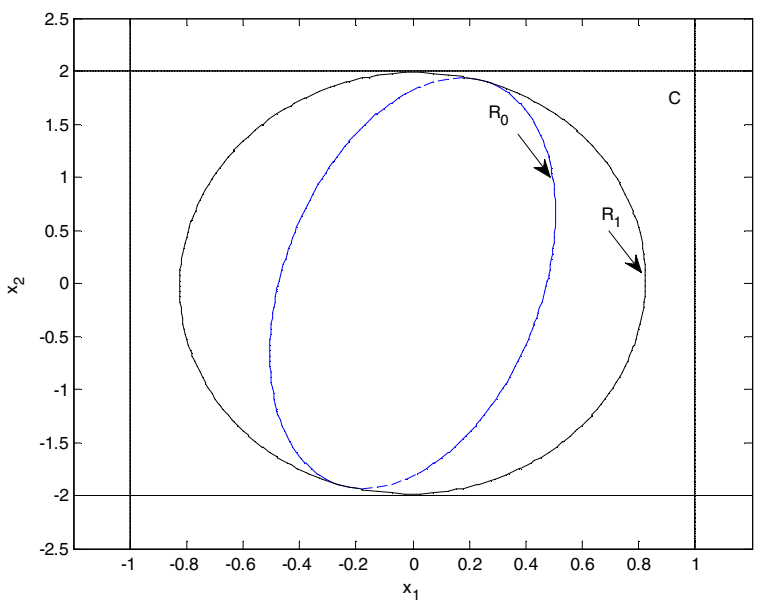

Figure 2. Outermost Lyapunov level for theorem 2 (R1) and conditions of [4] (R0) applied to example (39). 
Fig. 1 shows the highest quadratic Lyapunov level corresponding to this example. Four trajectories of the controlled model via a non-PDC control law have been included which show the convergence towards the origin.

In comparison, the classical approaches [4] gives the outermost Lyapunov level reduced, showed Fig. 2.

Example 2: Consider the following TS model with:

$\dot{x}(t)=A_{z} x(t)+B_{z} u(t)=\sum_{i=1}^{2} h_{i}(z(t))\left(A_{i} x(t)+B_{i} u(t)\right)$

with $A_{1}=\left[\begin{array}{ll}3.6 & -1.6 \\ 6.2 & -4.3\end{array}\right], \quad A_{2}=\left[\begin{array}{ll}-a & -1.6 \\ 6.2 & -4.3\end{array}\right], \quad B_{1}=\left[\begin{array}{c}-0.45 \\ -3\end{array}\right]$,

$B_{2}=\left[\begin{array}{l}-b \\ -3\end{array}\right] \quad, \quad z_{1}(t)=x_{1}(t) \quad, \quad h_{1}=w_{0}^{1}=\frac{1-\sin x_{1}}{2} \quad$ and

$h_{2}=w_{1}^{1}=\frac{1+\sin x_{1}}{2} \quad$ defined in the compact set

$C=\left\{x:\left|x_{i}\right| \leq \pi / 2\right\}, i=1,2$.

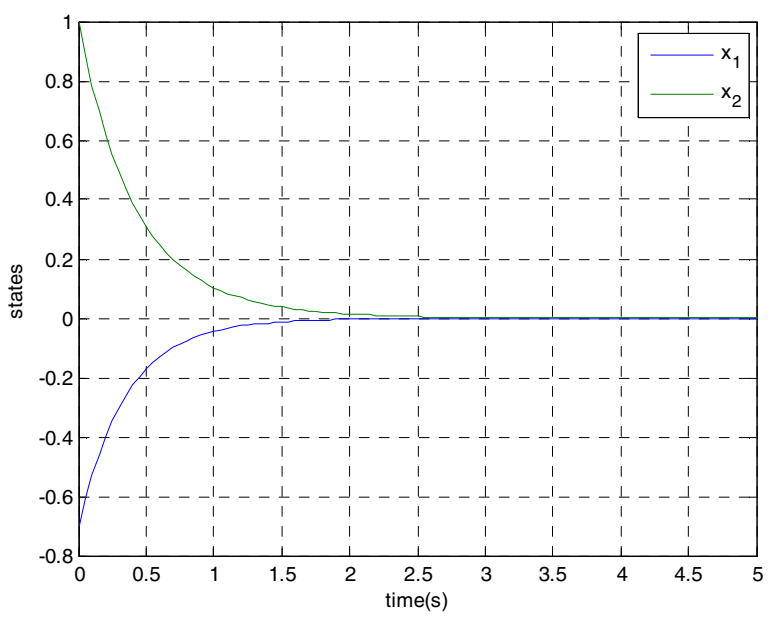

Figure 3. Time evolution of the states under theorem 3 scheme.

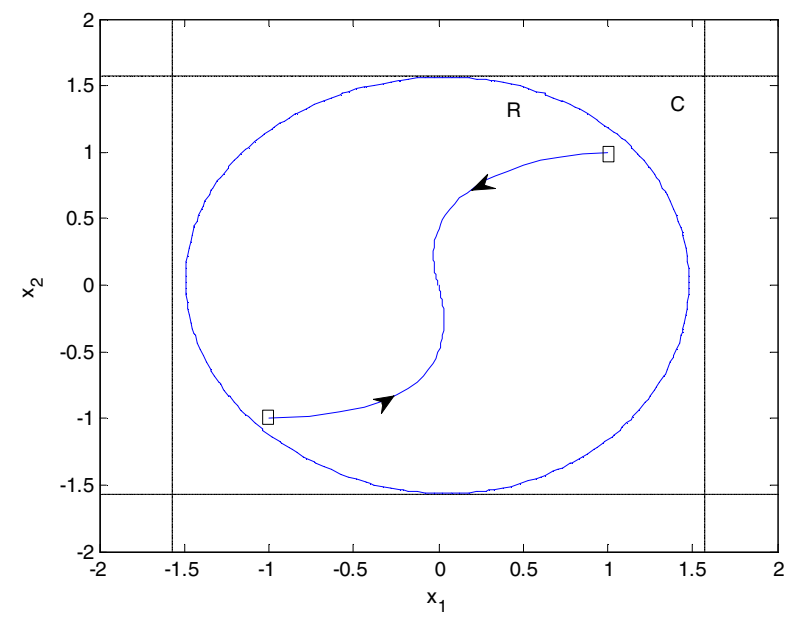

Figure 4. The outermost Lyapunov level for theorem 3 applied to example 2.
With $a=0$ and $b=2$, there are no result for a quadratic function neither [4], nor theorems $1 \& 2$. A non-PDC controller of the form (22) under a non-quadratic Lyapunov function as (21) is considered with $\varepsilon=1, \lambda_{x}^{2}=\frac{\pi^{2}}{2}, \lambda_{k}^{2}=0.25$ and $\beta=0.2$. Theorem 3 provides a feasible solution. The simulation has been performed from initial conditions $x(0)=\left[\begin{array}{ll}-0.7 & 1\end{array}\right]^{T}$. The time evolution of the states is shown in Fig. 3. The outermost Lyapunov level is also plotted Fig. 4.

\section{CONCLUSIONS}

A new scheme for non-PDC controller design for continuous-time nonlinear models in the TS form has been presented. The basis is to "cut" the link between the Lyapunov function and the controller via Finsler's Lemma, thus providing additional flexibility to the design. Quadratic cases as well as former non-quadratic approaches have been included through a suitable choice of the MFs; these choices allow the well-known problem of handling the time-derivatives of the MFs to be circumvented in a simple way. Examples illustrating the effectiveness of this technique have been provided.

\section{REFERENCES}

[1] T. Takagi and M. Sugeno, "Fuzzy identification of systems and its application to modeling and control", IEEE Trans. on System Man and Cybernetics, Vol.15(1), pp116-132. 1985.

[2] T. Taniguchi, K. Tanaka, H.O. Wang, "Model construction, rule reduction and robust compensation for generalized form of TakagiSugeno fuzzy systems", IEEE Trans. on Fuzzy Systems, Vol.9(4), pp525-537. 2001.

[3] K. Tanaka and H.O. Wang, Fuzzy control systems design and analysis. A linear matrix inequality approach. John Wiley and Sons, New York, USA. 2001.

[4] H.O. Wang, K. Tanaka, M. Griffin, "An approach to fuzzy control of nonlinear systems: Stability and Design Issues", IEEE Trans. on Fuzzy Systems, Vol.4(1), pp14-23. 1996.

[5] G. Feng, "A Survey on Analysis and Design of Model-Based Fuzzy Control Systems", IEEE Trans. on Fuzzy Systems, 14 (5): 676-697, 2006.

[6] S. Boyd, L. El Ghaoui, E. Féron, V. Balakrishnan, Linear matrix inequalities in system and control theory. Studies in Applied Mathematics; Philadelphia. 1994.

[7] A. Sala, T.M. Guerra, R. Babuska, "Perspectives of fuzzy systems and control”, Fuzzy Sets \& Systems, Vol.156, pp432-444. 2005.

[8] K. Guelton, T. Bouarar, and N. Manamanni, "Robust dynamic output feedback fuzzy Lyapunov stabilization of Takagi-Sugeno systems-A descriptor redundancy approach", Fuzzy Sets \& Systems, vol. 160 (19), 2009, pp. 2796-2811.

[9] K. Tanaka, H. Yoshida, H. Ohtake, and H.O. Wang. "A sum of squares approach to modeling and control of nonlinear dynamical systems with polynomial fuzzy systems", IEEE Trans. on Fuzzy Systems, Vol. 17(4), pp 911-922, 2009.

[10] M. Johansson, A. Rantzer, K. Arzen, "Piecewise quadratic stability of fuzzy systems". IEEE Trans. on Fuzzy Systems, Vol.7, pp713-722,1999.

[11] G. Feng, "Stability Analysis of Discrete-Time Fuzzy Dynamic Systems Based on Piecewise Lyapunov functions". IEEE Trans. on Fuzzy Systems, Vol. 12 (1), pp 22-28, 2004.

[12] X. Liu and Q. Zhang, "New approaches to controller designs based on fuzzy observers for T-S fuzzy systems via LMI", Automatica, 39 (9): 1571-1582. 
[13] Y. Blanco, W. Perruquetti, P.Borne, "Stability and stabilization of nonlinear systems and Tanaka-Sugeno fuzzy models", in European Control Conference, Lisbonne, Portugal, 2001.

[14] K. Tanaka, T. Hori, H.O. Wang, "A multiple Lyapunov function approach to stabilization of fuzzy control systems", IEEE Trans. on Fuzzy Systems, Vol. 11 (4), pp 582-589, 2003.

[15] B.J. Rhee, S. Won, "A new fuzzy Lyapunov function approach for a Takagi-Sugeno fuzzy control system design", Fuzzy Sets \& Systems, Vol. 157(9), pp1211-1228, 2006.

[16] T.M. Guerra and L. Vermeiren, "LMI-based relaxed non-quadratic stabilization conditions for nonlinear systems in Takagi-Sugeno's form", Automatica, Vol. 40(5), pp823-829. 2004.

[17] B.C. Ding, H.X. Sun, P. Yang, "Further studies on LMI-based relaxed stabilization conditions for nonlinear systems in Takagi-Sugeno's form". Automatica Vol. 42, pp503-508, 2006.

[18] T.M. Guerra, A. Kruszewski, M. Bernal, "Control Law Proposition for the Stabilization of Discrete Takagi-Sugeno Models". IEEE Trans. on Fuzzy Systems, 17 (3), 724-731, 2009.

[19] A. Sala, C. Ariño, "Asymptotically necessary and sufficient conditions for stability and performance ixn fuzzy control: Applications of Polya's theorem" Fuzzy Sets and Systems, Vol. 158, (24), pp 2671-2686, 2007.

[20] A. Sala and C. Ariño. Relaxed stability and performance conditions for Takagi-Sugeno fuzzy systems with knowledge on membership-function overlap. IEEE Trans. SMC(B), 37(3):727-732, 2007.

[21] M. Bernal, T.M. Guerra, A. Kruszewski, "A membership-functiondependent approach for stability analysis and controller synthesis of Takagi-Sugeno models". Fuzzy Sets \& Systems. Vol. 160 (19), pp 27762795, 2009 .

[22] M. Bernal and T.M. Guerra, "Generalized non-quadratic stability of continuous-time Takagi-Sugeno models", IEEE Trans. on Fuzzy Systems, vol. 18 (4), 2010, pp 815-822.

[23] M. Bernal, T.M. Guerra, and A. Jaadari, "Non-quadratic stabilization of Takagi-Sugeno models: a local point of view"; Proc. of FUZZ-IEEE Conference, Barcelona, Spain, 2010, pp. 2375-2380.

[24] H.D. Tuan, P. Apkarian, T. Narikiyo, and Y. Yamamoto. "Parameterized linear matrix inequality techniques in fuzzy control system design". IEEE Trans. on Fuzzy Systems, 9(2):324-332, 2001.

[25] H. Khalil, Nonlinear Systems, third edition. Prentice Hall, New Jersey, USA. 2002.

[26] T.M. Guerra and M. Bernal, "A way to escape from the quadratic framework", in Proc. FUZZ-IEEE Conference, Jeju, Korea, 2009.
[27] T.M Guerra, A. Kruszewski, J. Lauber, "Discrete Takagi-Sugeno models for control: Where are we?", Annual Reviews in Control, 33: 37-47, 2009.

[28] T.M Guerra, M. Bernal, A. Kruszewski, M. Afroun, "A way to improve results for the stabilization of continuous-time fuzzy descriptor models", in Proc. 46th Control and Decision Conference, pp 5960 - 5964, New Orleans, LA. 2007.

[29] G. Feng, C.L. Chen, D. Sun, Y. Zhu, "Hळ controller synthesis of fuzzy dynamic systems based on piecewise Lyapunov functions and bilinear matrix inequalities” . IEEE Trans. On Fuzzy Systems, Vol. 13 (1), pp 94-103, 2005.

[30] M. Bernal, P. Hušek, V. Kučera, "Non quadratic stabilization of continuous-time systems in the Takagi-Sugeno form", Kybernetika, vol. 42 (6), pp. 665-672, 2006.

[31] M. Bernal, A. Sala, T.M. Guerra, A. Jaadari, "Stability analysis of polynomial fuzzy models via polynomial fuzzy Lyapunov functions", Fuzzy Sets and Systems, 185 (1): 5-14, 2011.

[32] L. Mozelli, R. Palhares, G.S.C. Avellar, "A systematic approach to improve multiple Lyapunov function stability and stabilization conditions for fuzzy systems", Information Sciences, Vol. 179(8), pp 1149-1162.

[33] T.M. Guerra, A. Jaadari, J. Pan, A. Sala, "Some Refinements for Nonquadratic Stabilization of continuous TS Models", In Proc. FUZZIEEE Conf. 2011, pp 329-333, Taipei, Taiwan, 2011.

[34] M.C. de Oliveira, R.E. Skelton, "Stability tests for constrained linear systems. In perspectives in Robust Control", Lecture Notes in Control and Information Sciences, S.O. Reza Moheimani Ed. Springer-Verlag 268, 241-257, 2001.

[35] R.C.L.F. Oliveira, M.C. de Oliveira, P.L.D. Peres, "Robust state feedback LMI methods for continuous-time linear systems: discussions, exyensions and numerical comparisons", IEEE Multi-Conf Systems \& Control, Denver, CO, USA, 2011

[36] Z. Lendek, T.M. Guerra, R. Babuska, "On non-PDC local observers for TS fuzzy systems", In Proc. of FUZZ-IEEE Conference, Barcelona, Spain, 2010, pp. 2436-2442.

[37] J. Pan, T.M. Guerra, S.M. Fei, A. Jaadari, "Nonquadratic stabilization of continuous T-S fuzzy models : LMI solution for a local approach", IEEE Trans. On Fuzzy Systems, 2012 (To appear) 